

\title{
Diameter-modulated ferromagnetic CoFe nanowires
}

\section{A. S. Esmaeily, M. Venkatesan, Asra S. Razavian, and J. M. D. Coey}

Citation: Journal of Applied Physics 113, 17 A327 (2013); doi: 10.1063/1.4794722

View online: http://dx.doi.org/10.1063/1.4794722

View Table of Contents: http://scitation.aip.org/content/aip/journal/jap/113/17?ver=pdfcov

Published by the AIP Publishing

\section{Articles you may be interested in}

Off-axis electron holography of ferromagnetic multilayer nanowires

J. Appl. Phys. 116, 023902 (2014); 10.1063/1.4887488

Structural and magnetic characterization of as-prepared and annealed FeCoCu nanowire arrays in ordered anodic aluminum oxide templates

J. Appl. Phys. 115, 133904 (2014); 10.1063/1.4870289

Tuning the magnetization reversal process of $\mathrm{FeCoCu}$ nanowire arrays by thermal annealing

J. Appl. Phys. 114, 043908 (2013); 10.1063/1.4816479

Enhanced magnetic performance of metal-organic nanowire arrays by $\mathrm{FeCo} /$ polypyrrole co-electrodeposition J. Appl. Phys. 113, 17B908 (2013); 10.1063/1.4800835

Ordered Ni nanowire tip arrays sticking out of the anodic aluminum oxide template

J. Appl. Phys. 97, 064303 (2005); 10.1063/1.1846942

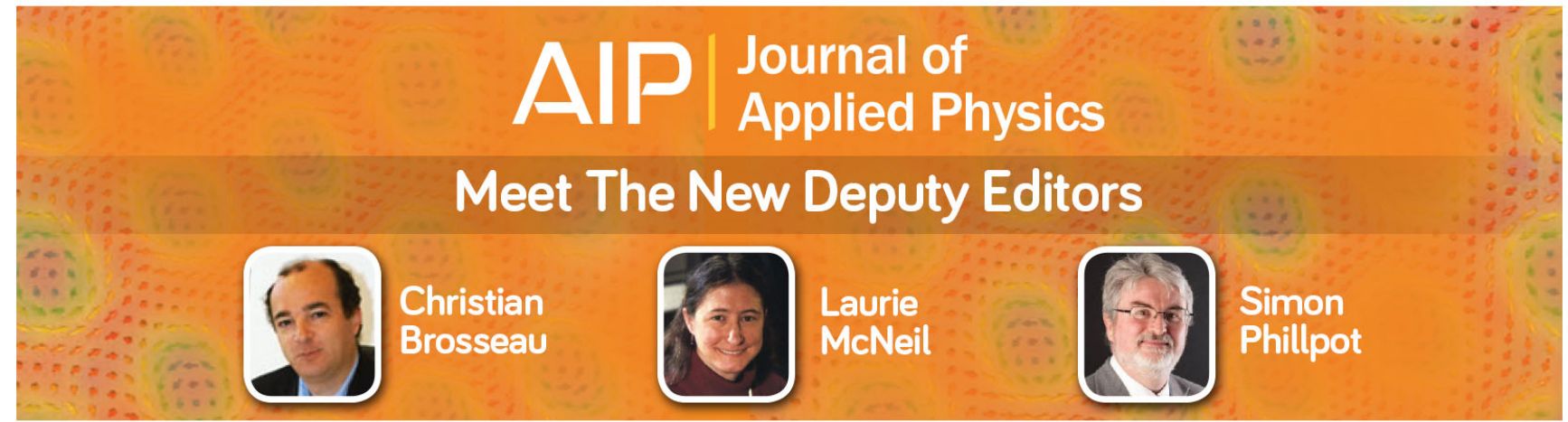




\title{
Diameter-modulated ferromagnetic CoFe nanowires
}

\author{
A. S. Esmaeily, ${ }^{1, a)}$ M. Venkatesan, ${ }^{1}$ Asra S. Razavian, ${ }^{1,2}$ and J. M. D. Coey ${ }^{1}$ \\ ${ }^{1}$ School of Physics and CRANN, Trinity College, Dublin 2, Ireland \\ ${ }^{2}$ Institute of Nanoscience and Nanotechnology, University of Kashan, Iran
}

(Presented 15 January 2013; received 5 November 2012; accepted 30 November 2012; published online 27 March 2013)

\begin{abstract}
Highly ordered nanoporous anodic aluminium oxide templates with uniform or periodically modulated pore diameters have been fabricated by the hard-pulse anodization technique. Straight and diameter-modulated CoFe nanowires with diameters of $60 \mathrm{~nm}$ and $60 / 120 \mathrm{~nm}$, respectively, were AC-pulse electrodeposited into the templates. A comparison of magnetisation curves of the two types of nanowires show a typical coercivity $\left(\mu_{0} H_{\mathrm{c}}\right)$ of $74 \mathrm{mT}$ and squareness (SQ) of $92 \%$ for the modulated wires, which are significantly squarer but have $12 \%$ lower coercivity compared to the $60 \mathrm{~nm}$ straight $\mathrm{CoFe}$ wires $\left(\mu_{0} H_{\mathrm{c}}=84 \mathrm{mT}, \mathrm{SQ}=60 \%\right)$. The difference is attributed to magnetostatic interactions between the segments due to the stray field of the modulated wires. Diameter modulation is an attractive way of producing soft magnetic nanowires with a controllable hysteresis loop shape. The time required for the nanoporous template fabrication by hard-pulse anodization is typically one tenth of that needed for conventional template fabrication methods, hence making it suitable for large-scale nanowire production. (c) 2013 American Institute of Physics. [http://dx.doi.org/10.1063/1.4794722]
\end{abstract}

\section{INTRODUCTION}

During past two decades, template-based fabrication methods ${ }^{1}$ have become one of the most effective ways of fabricating arrays of nanowires, ${ }^{2}$ nanoparticles, and nanotubes. $^{3}$ In this way, many different porous templates like track etched mica, ${ }^{4}$ track etched polycarbonate, ${ }^{5}$ or anodized aluminum $^{6}$ have been used. But, without a doubt, after the work of Masuda and Fukuda in 1995 on two step mild anodization of aluminum, ${ }^{6}$ anodic aluminium oxide (AAO) templates, have become popular to fabricate highly ordered arrays of one- and zero-dimensional nanostructures. ${ }^{7}$ During the first step lasting about a day or more, aluminum is anodized at a voltage of typically $40 \mathrm{~V}$ in an acidic electrolyte, and the resulting nanoporous oxide layer is removed by wet etching. In the second step lasting about an hour, the patterned aluminum is reanodized under the same conditions to form an ordered nanoporous aluminum oxide template. Twostep anodization is a very time-consuming process. Recently, Lee et al. ${ }^{8}$ introduced a "hard" anodization technique, which is based on a new self-ordering regime of aluminium during anodization at voltages generally above $100 \mathrm{~V}$. Although the alignment and speed of pore formation are much higher for hard anodization than for conventional two step mild anodization, this template fabrication approach has been little used for nanowire fabrication, except in some recent work. ${ }^{9}$ Modifying the electrodeposition conditions ${ }^{10}$ or electrolyte composition $^{11}$ are traditionally the only ways to tune magnetic properties of nanowires electrodeposited into AAO templates.

\footnotetext{
a) Author to whom correspondence should be addressed. Electronic mail: esmaeila@tcd.ie.
}

Here we report a new method for producing magnetic nanowires where the whole fabrication process time is cut to about one tenth of that needed with conventional two-step anodization. Furthermore, the method allows us to tune the magnetic properties of the nanowires formed in the alumina template.

\section{EXPERIMENT}

Aluminium discs (high purity $99.999 \%$ ) of $12 \mathrm{~mm}$ diameter, $0.25 \mathrm{~mm}$ thick are ultrasonically cleaned in acetone for $3 \mathrm{~min}$, electropolished in a 1:4 volume mixture of perchloric acid and ethanol for $3 \mathrm{~min}$ under a current density of $100 \mathrm{~mA} / \mathrm{cm}^{2}$, and rinsed in deionized water. Discs are then anodized at $40 \mathrm{~V}$ in an aqueous solution of $0.3 \mathrm{M}$ oxalic acid at $0{ }^{\circ} \mathrm{C}$ for $10 \mathrm{~min}$ to produce a protective layer of aluminum oxide about $500 \mathrm{~nm}$ thick at the surface of the disc, which suppresses breakdown and burning effects during subsequent hard-pulse anodization. ${ }^{8}$ The anodization voltage was then increased to $120 \mathrm{~V}$ at a rate of $0.5 \mathrm{Vs}^{-1}$ while intensely stirring the electrolyte. This is an essential transition step between mild and hard anodization self-ordering regimes. The voltage is held at $120 \mathrm{~V}$ for $1200 \mathrm{~s}$ to allow alignment of nanochannels. To produce modulated-channels in the alumina template, a set of consecutive hard anodization potential pulses of $140 \mathrm{~V}$ and $160 \mathrm{~V}$ were applied for 40 and $10 \mathrm{~s}$, respectively, at $80 \mathrm{~s}$ intervals. Pulses were repeated 50 times, producing long nanopores of AAO with modulated segments, a few hundred nanometers in length with the diameters of 60 and $120 \mathrm{~nm}$, respectively. The anodization voltage profile can be seen in Fig. 1. To promote thinning of the alumina barrier layer necessary to allow AC-pulse electrodeposition of the samples, the voltage was exponentially decreased from $120 \mathrm{~V}$ to $12 \mathrm{~V}$. Straight templates were 


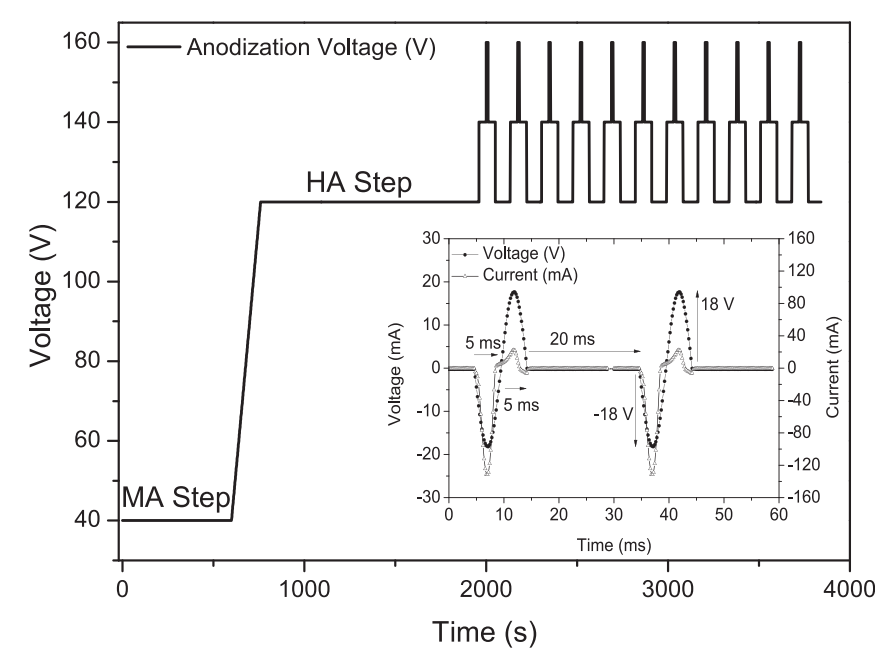

FIG. 1. Anodization potential showing the hard anodization pulses used to produce the diameter modulated templates and (inset) AC-pulse electrodeposition profile; $18 / 18 \mathrm{~V}$ oxidation/reduction potential each with $5 \mathrm{~ms}$ duration and $20 \mathrm{~ms}$ off-time between pulses.

fabricated by same procedure at $120 \mathrm{~V}$, without the extra anodization pulses. Total time for the template fabrication for both straight and modulated nanowires was around $2 \mathrm{~h}$.

A sinusoidal AC wave of $18 \mathrm{~V}$ was then used to deposit the nanowires into the templates from an electrolyte containing $0.3 \mathrm{M} \mathrm{FeSO}_{4} .7 \mathrm{H}_{2} \mathrm{O}, 0.3 \mathrm{M} \mathrm{CoSO}_{4} .7 \mathrm{H}_{2} \mathrm{O}, 1 \mathrm{~g} / \mathrm{l}$ ascorbic acid, and $45 \mathrm{~g} / \mathrm{l}$ boric acid at $\mathrm{pH} \sim 5,30^{\circ} \mathrm{C}$ for $30 \mathrm{~min}$. $\mathrm{CoFe}$ nanowires were $\mathrm{AC}$-pulse electrodeposited into the templates using $18 / 18 \mathrm{~V}$ oxidation/reduction sinusoidal pulses with $20 \mathrm{~ms}$ off-time between pulses. Fig. 1 inset shows a typical deposition voltage/current curve. The magnetic properties of as-prepared diameter-modulated and straight nanowires were measured by SQUID at room temperature. The structural and morphological characterization of the nanowires was carried out by scanning electron microscopy (SEM) and X-ray diffractometry (XRD). Energy dispersive spectroscopy (EDS) analysis was used to examine the composition of the nanowires.

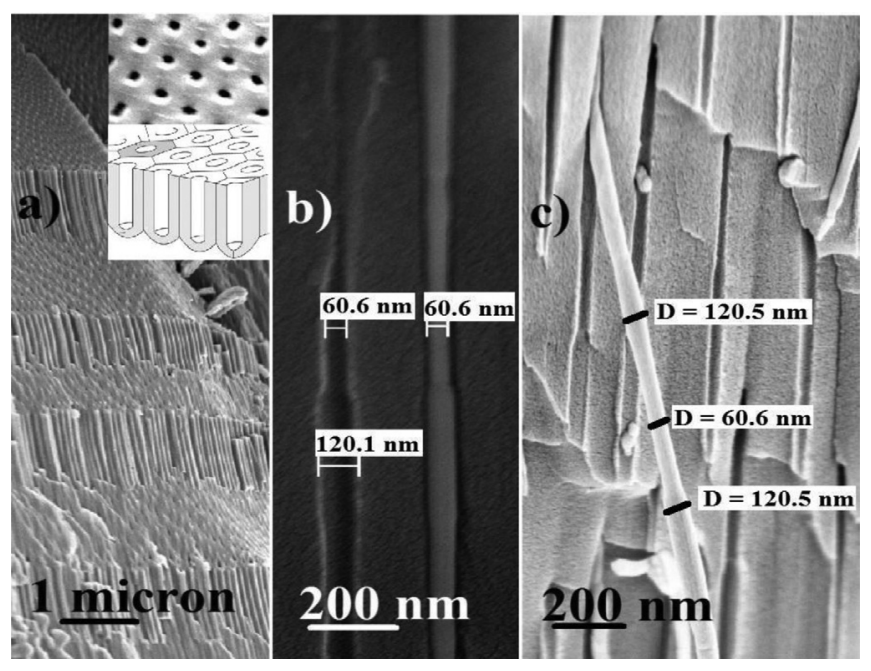

FIG. 2. (a) A typical diameter-modulated alumina template with seven segments. (b) Diameter-modulated CoFe nanowires inside template and (c) released from template.

\section{RESULTS AND DISCUSSION}

The alumina templates with straight $60 \mathrm{~nm}$ or diametermodulated $60 / 120 \mathrm{~nm}$ nanopores prepared by the above method were highly ordered (Fig. 2(a) top inset). The EDS analysis confirmed formation of straight and diametermodulated $\mathrm{Co}_{0.5} \mathrm{Fe}_{0.5}$ nanowires in AAO templates as expected since the electrodeposition rate of $\mathrm{Fe}^{2+}$ ions was almost the same as that of $\mathrm{Co}^{2+}$ ions in the electrolyte used. ${ }^{9}$ Both sets of nanowire arrays showed preferential (110) growth orientation along the wire.

Fig. 2(a) shows a typical diameter-modulated AAO template. XRD patterns of templates containing diametermodulated and straight nanowires show a bcc structure with lattice parameter $\mathrm{a}_{0}=285 \mathrm{pm}$ and (110) orientation. Figs. 2(b) and 2(c) show the SEM images of CoFe nanowires grown in a diameter-modulated AAO template with alternating $60 \mathrm{~nm}$ and $120 \mathrm{~nm}$ segments.

Figs. 3(a) and 3(b) compares hysteresis behaviour of straight and diameter-modulated CoFe nanowire arrays. The field is applied either parallel or perpendicular to the alumina template. The squareness SQ, defined as the ratio of remanence to saturation magnetization of diametermodulated CoFe nanowires, is significantly improved compared with that of the straight wires. A typical coercivity and squareness of $74 \mathrm{mT}$ and $92 \%$ were observed for the modulated wires, compared with $\mu_{0} H_{\mathrm{c}}=84 \mathrm{mT}$ and SQ $=60 \%$ for the straight $60 \mathrm{~nm}$ CoFe wires. The data suggest magnetostatic interactions between the segments with a substantial stray field, which is absent in longitudinally magnetized wires of uniform diameter. There is no demagnetizing field acting on a single long wire magnetized along its axis. However, when the wires are assembled in a template, the resulting nanowire array considered as the summation of all the wires in the nanopores, produces a demagnetising field like a piece of uniformly magnetized material. Since the demagnetizing field will be proportional to the averaged magnetization, it will be approximately ${ }^{12}$

$$
H_{\mathrm{d}}=-f M_{\mathrm{s}}
$$

where $M_{\mathrm{s}}$ for the CoFe is $1.5 \mathrm{MAm}^{-1}$ and $f$ is the fill factor of the template, which counts only the material filled in the pores, since the rest of the volume is alumina. Each pore and the region surrounding it comprises an hexagonal cell. The ratio of the nanowire cross-section to the area of the hexagonal unit cell is the fill factor $f$. In the case of pores of radius $r$ with spacing $d, f=(2 / \sqrt{ } 3) \pi(r / d)^{2}$. For $\mathrm{r}=30 \mathrm{~nm}$ pores with a spacing of $250 \mathrm{~nm}, f=0.052$. Hence, $H_{\mathrm{d}}=-78 \mathrm{kAm}^{-1}$. This accounts for the shearing of the loop in Fig. 3(a).

In a modulated wire, the stray field acting on a central segment due to the magnetic charge on the rims of the wide segments is proportional to the magnetisation ${ }^{12}$

$$
H_{\mathrm{d}}=g M_{\mathrm{s}},
$$

where $g$ may be estimated as

$$
g=1.2\left(r_{1}{ }^{2}-r_{2}{ }^{2}\right) l_{1} /\left(l_{1}+l_{2}\right)^{3},
$$



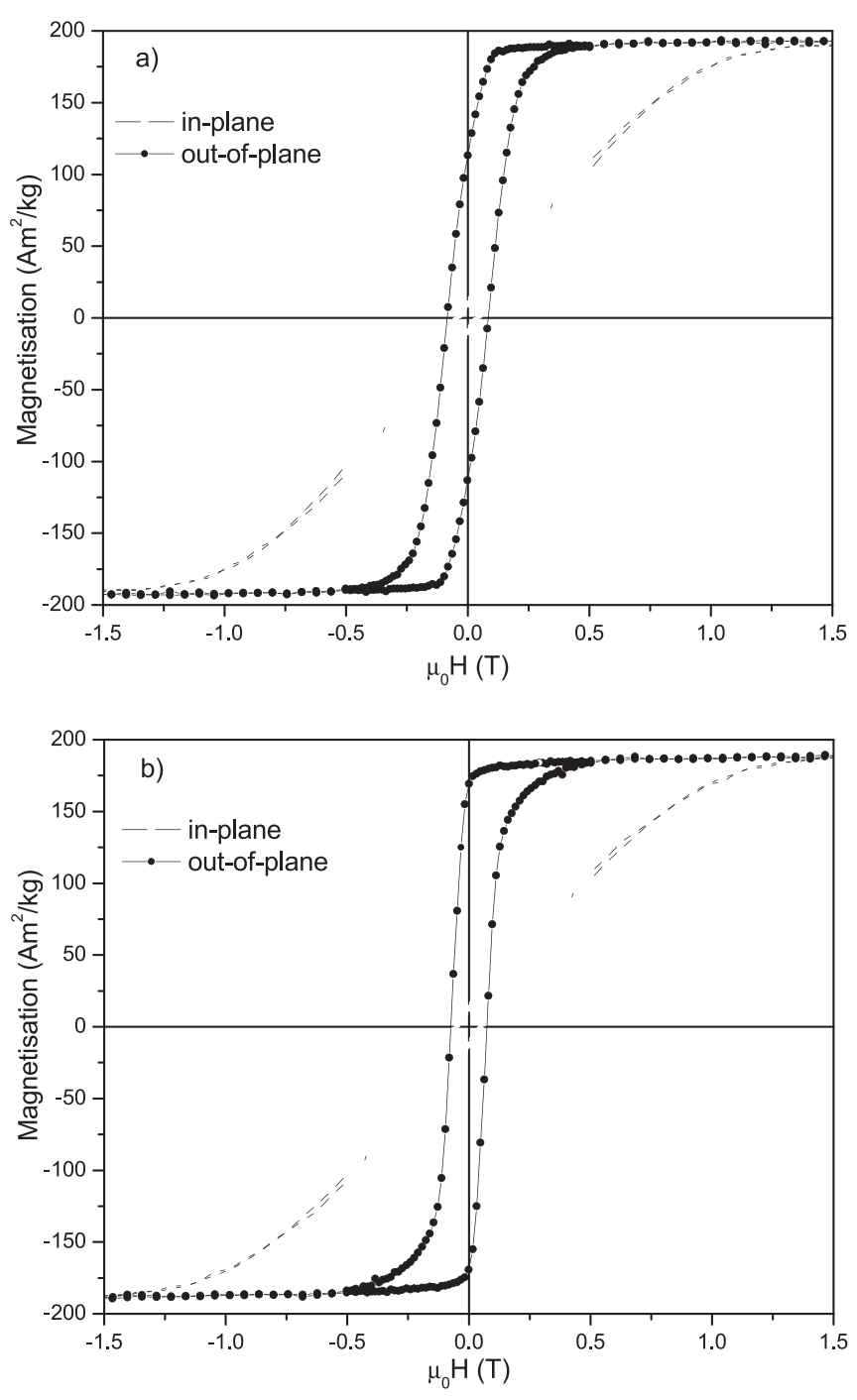

FIG. 3. Magnetisation curves of (a) $60 \mathrm{~nm}$ straight and (b) $60 / 120 \mathrm{~nm}$ diameter-modulated $\mathrm{CoFe}$ nanowires.

where $r_{1}$ and $r_{2}$ or $l_{1}$ and $l_{2}$ are the radii and length of the thick and thin segments, respectively. This expression gives the field at the centre of a line of magnetized rings, after removing the long wire of radius $r_{2}$, which makes no contribution. The factor 1.2 is $\sum\left(1 / \mathrm{n}^{3}\right)$ from the Fig. 2(b). Taking $r_{1}=60 \mathrm{~nm}, r_{2}=30 \mathrm{~nm}, l_{1}=l_{2}=300 \mathrm{~nm}$ gives $\mathrm{g}=0.005$. We see that with our experimental geometry, the stray field of the modulated wire can significantly reduce the magnitude of the demagnetizing field, which helps to account for the improved squareness of the loop. The coercivity of magnetic nanowires has been found to increase as the diameter of the wires decreases. ${ }^{9,13,14}$ In our modulated wires, compared to the straight wires of the smaller diameter, the wires have segments with wider diameters that tend to reduce the coercivity.

\section{CONCLUSION}

Hard pulse anodization is an advantageous method of preparing AAO templates for nanowire fabrication for two reasons: (1) The time required for the fabrication of templates by hard pulse anodization is much less than for the conventional two-step method. (2) The diameter of the pores can be modulated along their length. Diameter-modulated nanowires can be produced by electrodeposition into the pores. The soft magnetic properties of the wires can be tuned by modifying the diameter profile. In particular, hysteresis loops with improved squareness can be obtained. This is attributed to the local stray field in the hexagonal array; no such effect is expected for a cubic array, where the dipole sum is zero. Since the diametermodulated nanowires produce a characteristic pattern of stray field, depending on the thickness profile, they may be suitable for use as magnetic markers. The hard pulse anodization membrane preparation method opens the prospect of rapidly fabricating ferromagnetic nanowires with hysteresis loops of controllable shape, thereby introducing a new degree of freedom to magnetic nanowire fabrication.

\section{ACKNOWLEDGMENTS}

This work was partially supported by Science Foundation Ireland (SFI) and the EU FP7 NAMDIATREAM project (NMP-2009-LARGE-3, project no. 246479). Electron microscopy work was conducted in the CRANN Advanced Microscopy Laboratory.

${ }^{1}$ A. Huczko, Appl. Phys. A 70, 365 (2000).

${ }^{2}$ X. Xu and G. Zangari, J. Appl. Phys. 97, 10 A306 (2005).

${ }^{3}$ K. Yu, G. Ruan, Y. Ben, and J. Zou, Mater. Lett. 61, 97 (2007).

${ }^{4}$ L. Sun, C. L. Chien, and P. C. Searson, J. Mater. Sci. 35, 1097 (2000).

${ }^{5}$ C. Schönenberger, B. M. I. van der Zande, L. G. J. Fokkink, M. Henny, C. Schmid, M. Krüger, A. Bachtold, R. Huber, H. Birk, and U. Staufer, J. Phys. Chem. B 101, 5497 (1997).

${ }^{6}$ H. Masuda and K. Fukuda, Science 268, 1466 (1995).

${ }^{7}$ Zh. Luo, W. Yang, A. Peng, Y. Zeng, and J. Yao, Nanotechnology 20, 345601 (2009).

${ }^{8}$ W. Lee, R. Ji, U. Gösele, and K. Nielsch, Nature Mater. 5, 741 (2006).

${ }^{9}$ M. Almasi Kashi, A. Ramazani, and A. S. Esmaeily, IEEE Trans. Magn. 49, 1167 (2013).

${ }^{10}$ M. Almasi Kashi, A. Ramazani, S. Doudafkan, and A. S. Esmaeily, Appl. Phys. A 102, 761 (2011).

${ }^{11}$ A. Encinas, M. Demand, J.-M. George, and L. Piraux, IEEE Trans. Magn. 38, 2574 (2002)

${ }^{12}$ J. M. D. Coey, Magnetism and Magnetic Materials, (Cambridge University Press, 2010).

${ }^{13}$ Q. F. Liu, C. X. Gao, J. J. Xiao, and D. S. Xue, J. Magn. Magn. Mater. 260, 151 (2003).

${ }^{14}$ T. Shimizu, A. Aoki, Y. Tanaka, T. Terui, and S. Shingubara, Jpn. J. Appl. Phys. 50, 06GE01 (2011). 